Commun. Korean Math. Soc. 24 (2009), No. 3, pp. 415-424

DOI 10.4134/CKMS.2009.24.3.415

\title{
ON SOME CLASSES OF GENERALIZED QUASI-EINSTEIN MANIFOLDS
}

\author{
Absos Ali Shaikh and Shyamal Kumar Hui
}

\begin{abstract}
The object of the present paper is to study the generalized quasi-Einstein manifolds satisfying some conditions. Finally the existence of such manifolds is ensured by several interesting examples.
\end{abstract}

\section{Introduction}

The notion of quasi-Einstein manifolds was introduced by M. C. Chaki and R. K. Maity [1]. A Riemannian manifold $\left(M^{n}, g\right)(n>2)$ is said to be quasiEinstein manifold if its Ricci tensor $S$ of type $(0,2)$ is not identically zero and satisfies the following:

$$
S(X, Y)=\alpha g(X, Y)+\beta A(X) A(Y),
$$

where $\alpha, \beta$ are scalars of which $\beta \neq 0$ and $A$ is a nowhere vanishing 1-form defined by $g(X, \rho)=A(X)$ for all vector fields $X ; \rho$ being a unit vector field, called the generator of the manifold. An $n$-dimensional manifold of this kind is denoted by $(Q E)_{n}$. The scalars $\alpha, \beta$ are known as the associated scalars.

As a generalization of quasi-Einstein manifold, in [2], U. C. De and G. C. Ghosh introduced the notion of generalized quasi-Einstein manifold. A Riemannian manifold $\left(M^{n}, g\right)(n \geq 3)$ is said to be generalized quasi-Einstein manifold if its Ricci tensor $S$ of type $(0,2)$ is not identically zero and satisfies the following:

$$
S(X, Y)=\alpha g(X, Y)+\beta A(X) A(Y)+\gamma B(X) B(Y),
$$

where $\alpha, \beta, \gamma$ are scalars of which $\beta \neq 0, \gamma \neq 0$ and $A, B$ are nowhere vanishing 1-forms such that $g(X, \rho)=A(X), g(X, \mu)=B(X)$ for all vector fields $X$. The unit vectors $\rho$ and $\mu$ corresponding to the 1-forms $A$ and $B$ are orthogonal to each other. Also $\rho$ and $\mu$ are known as the generators of the manifold. Such an $n$-dimensional manifold is denoted by $G(Q E)_{n}$.

Received December 17, 2008; Revised May 5, 2009.

2000 Mathematics Subject Classification. 53B30, 53B50, 53C50, 53C80, 83D05.

Key words and phrases. generalized quasi-Einstein manifold, Ricci-pseudosymmetric manifold, Ricci-semisymmetric manifold, conformal curvature tensor, concircular curvature tensor, quasi-conformal curvature tensor, projective curvature tensor, conformally flat. 
The present paper deals with $G(Q E)_{n}$ satisfying some conditions. The paper is organized as follows. Section 2 is concerned with the preliminaries. Section 3 is devoted to the study of Ricci-pseudosymmetric $G(Q E)_{n}$. In Sections 47, we investigate the $G(Q E)_{n}(n>3)$ satisfying the conditions $C \cdot S=0$, $\tilde{C} \cdot S=0, W \cdot S=0$ and $P \cdot S=0$, where $C, \tilde{C}, W$ and $P$ respectively denote the conformal curvature tensor, concircular curvature tensor, quasi-conformal curvature tensor and projective curvature tensor. Then it is proved that in each of the case, either the associated scalars $\beta$ and $\gamma$ are equal or the curvature tensor $R$ satisfies a definite condition.

In Section 8, we study conformally flat Ricci-semisymmetric $G(Q E)_{n}(n>3)$ and it is shown that if in a conformally flat Ricci-semisymmetric $G(Q E)_{n}(n>$ 3), $\frac{r}{n-1}$ is not an eigenvalue of the Ricci-operator, then either the associated scalars $\beta$ and $\gamma$ are equal or the vector fields $\rho$ and $\mu$ corresponding to the 1-forms $A$ and $B$ are co-directional. The last section provides the existence of proper $G(Q E)_{n}$.

\section{Preliminaries}

In this section we will obtain some formulas of $G(Q E)_{n}$, which will be required in the sequel. Let $\left\{e_{i}: i=1,2, \ldots, n\right\}$ be an orthonormal frame field at any point of $G(Q E)_{n}$. Then setting $X=Y=e_{i}$ in (1.2) and taking summation over $i, 1 \leq i \leq n$, we obtain

$$
r=n \alpha+\beta+\gamma,
$$

where $r$ is the scalar curvature of the manifold.

Also, from (1.2), we have

$$
\begin{aligned}
& S(\rho, \rho)=\alpha+\beta, \\
& S(\mu, \mu)=\alpha+\gamma,
\end{aligned}
$$

and

$$
S(\rho, \mu)=0 .
$$

Let $Q$ be the Ricci-operator, i.e., $g(Q X, Y)=S(X, Y)$ for all $X, Y$.

\section{Ricci-pseudosymmetric $G(Q E)_{n}$}

An $n$-dimensional Riemannian manifold $\left(M^{n}, g\right)$ is called Ricci-pseudosymmetric [4] if the tensors $R \cdot S$ and $Q(g, S)$ are linearly dependent, where

$$
\begin{gathered}
(R(X, Y) \cdot S)(Z, U)=-S(R(X, Y) Z, U)-S(Z, R(X, Y) U), \\
Q(g, S)(Z, U ; X, Y)=-S\left(\left(X \wedge_{g} Y\right) Z, U\right)-S\left(Z,\left(X \wedge_{g} Y\right) U\right),
\end{gathered}
$$

$$
\left(X \wedge_{g} Y\right) Z=g(Y, Z) X-g(X, Z) Y
$$

for all vector fields $X, Y, Z, U$ of $M, R$ denotes the curvature tensor of $M$. 
Then $\left(M^{n}, g\right)$ is Ricci-pseudosymmetric if and only if

$$
(R(X, Y) \cdot S)(Z, U)=L_{S} Q(g, S)(Z, U ; X, Y)
$$

holds on $U_{s}=\left\{x \in M: S \neq \frac{r}{n} g\right.$ at $\left.x\right\}$, where $L_{S}$ is some function on $U_{S}$. If $R \cdot S=0$, then $M^{n}$ is called Ricci-semisymmetric. Every Ricci-semisymmetric manifold is Ricci-pseudosymmetric but the converse is not true [4].

In [2] De and Ghosh studied a Ricci-semisymmetric $G(Q E)_{n}$. We now consider a Ricci-pseudosymmetric $G(Q E)_{n}$. Then, from (3.1)-(3.4), we can write

$$
\begin{aligned}
& S(R(X, Y) Z, U)+S(Z, R(X, Y) U) \\
=\quad & L_{S}[g(Y, Z) S(X, U)-g(X, Z) S(Y, U) \\
+ & g(Y, U) S(X, Z)-g(X, U) S(Y, Z)] .
\end{aligned}
$$

Using (1.2) in (3.5), we get

$$
\begin{aligned}
& \beta[A(R(X, Y) Z) A(U)+A(Z) A(R(X, Y) U)] \\
& +\gamma[B(R(X, Y) Z) B(U)+B(Z) B(R(X, Y) U)] \\
= & L_{S}[\beta\{g(Y, Z) A(X) A(U)-g(X, Z) A(Y) A(U)+g(Y, U) A(X) A(Z) \\
& -g(X, U) A(Y) A(Z)\}+\gamma\{g(Y, Z) B(X) B(U) \\
& -g(X, Z) B(Y) B(U)+g(Y, U) B(X) B(Z)-g(X, U) B(Y) B(Z)\}] .
\end{aligned}
$$

Setting $Z=\rho$ and $U=\mu$ in (3.6), we get

$$
(\gamma-\beta)\left[R(X, Y, \rho, \mu)-L_{S}\{A(Y) B(X)-A(X) B(Y)\}\right]=0,
$$

which yields either $\beta=\gamma$ or

$$
R(X, Y, \rho, \mu)=L_{S}\{A(Y) B(X)-A(X) B(Y)\} .
$$

Hence we can state the following:

Theorem 3.1. In a Ricci-pseudosymmetric $G(Q E)_{n}(n>3)$, either the associated scalars $\beta$ and $\gamma$ are equal or the curvature tensor $R$ of the manifold satisfies the relation (3.8).

Corollary 3.1. In a Ricci-semisymmetric $G(Q E)_{n}(n>3)$, the associated scalars $\beta$ and $\gamma$ are equal [2].

\section{4. $G(Q E)_{n}(n>3)$ satisfying the condition $C \cdot S=0$}

The Weyl conformal curvature tensor $C$ of type $(1,3)$ of an $n$-dimensional Riemannian manifold $\left(M^{n}, g\right)(n>3)$ is defined by [3]

$$
\begin{aligned}
C(X, Y) Z= & R(X, Y) Z-\frac{1}{n-2}[S(Y, Z) X-S(X, Z) Y \\
& +g(Y, Z) Q X-g(X, Z) Q Y] \\
& +\frac{r}{(n-1)(n-2)}\{g(Y, Z) X-g(X, Z) Y\} .
\end{aligned}
$$


We now consider a $G(Q E)_{n}(n>3)$ satisfying the condition $C \cdot S=0$. Then we have

$$
S(C(X, Y) Z, U)+S(Z, C(X, Y) U)=0 .
$$

Using (1.2) in (4.2), we obtain

$$
\begin{aligned}
& \beta[A(C(X, Y) Z) A(U)+A(Z) A(C(X, Y) U)] \\
& +\gamma[B(C(X, Y) Z) B(U)+B(Z) B(C(X, Y) U)]=0 .
\end{aligned}
$$

Setting $Z=\rho$ and $U=\mu$ in (4.3), we get

$$
(\gamma-\beta) C(X, Y, \rho, \mu)=0 .
$$

From (4.4), it follows that either $\beta=\gamma$ or

$$
C(X, Y, \rho, \mu)=0,
$$

which further yields

$$
\begin{aligned}
R(X, Y, \rho, \mu)= & \frac{1}{n-2}[A(Q Y) B(X)-A(X) B(Q Y) \\
& +A(Y) B(Q X)-A(Q X) B(Y)] \\
& -\frac{r}{(n-1)(n-2)}\{A(Y) B(X)-A(X) B(Y)\} .
\end{aligned}
$$

Hence we can state the following:

Theorem 4.1. If a $G(Q E)_{n}(n>3)$ satisfies the condition $C \cdot S=0$, then either the associated scalars $\beta$ and $\gamma$ are equal or the curvature tensor $R$ of the manifold satisfies the property (4.5).

\section{5. $G(Q E)_{n}(n>3)$ satisfying the condition $\tilde{C} \cdot S=0$}

The concircular curvature tensor $\tilde{C}$ of type $(1,3)$ of an $n$-dimensional Riemannian manifold $\left(M^{n}, g\right)(n>3)$ is defined by [3]

$$
\tilde{C}(X, Y) Z=R(X, Y) Z-\frac{r}{n(n-1)}[g(Y, Z) X-g(X, Z) Y]
$$

for any vector fields $X, Y, Z \in \chi(M)$. Let us consider a $G(Q E)_{n}(n>3)$ satisfying the condition $\tilde{C} \cdot S=0$. Then we have

$$
S(\tilde{C}(X, Y) Z, U)+S(Z, \tilde{C}(X, Y) U)=0 .
$$

By virtue of (1.2), it follows from (5.2) that

$$
\begin{aligned}
& \beta[A(\tilde{C}(X, Y) Z) A(U)+A(Z) A(\tilde{C}(X, Y) U)] \\
& +\gamma[B(\tilde{C}(X, Y) Z) B(U)+B(Z) B(\tilde{C}(X, Y) U)]=0 .
\end{aligned}
$$

Putting $Z=\rho$ and $U=\mu$ in (5.3), we get

$$
(\gamma-\beta)\left[R(X, Y, \rho, \mu)-\frac{r}{n(n-1)}\{A(Y) B(X)-A(X) B(Y)\}\right]=0 .
$$

This leads to the following: 
Theorem 5.1. In a $G(Q E)_{n}(n>3)$ with $\tilde{C} \cdot S=0$, either the associated scalars $\beta$ and $\gamma$ are equal or the curvature tensor $R$ of the manifold satisfies the following property

$$
R(X, Y, \rho, \mu)=\frac{r}{n(n-1)}\{A(Y) B(X)-A(X) B(Y)\} .
$$

6. $G(Q E)_{n}(n>3)$ satisfying the condition $W \cdot S=0$

In 1968, Yano and Sawaki [5] defined and studied a curvature tensor $W$ of type $(1,3)$ which includes both the conformal curvature tensor $C$ and the concircular curvature tensor $\tilde{C}$ as special cases and is called quasi-conformal curvature tensor. The quasi-conformal curvature tensor $W$ of type $(1,3)$ of a manifold $\left(M^{n}, g\right)(n>3)$ is defined by

$$
W(X, Y) Z=-(n-2) b C(X, Y) Z+[a+(n-2) b] \tilde{C}(X, Y) Z,
$$

where $a$ and $b$ are arbitrary constants not simultaneously zero. In particular, if $a=1, b=0$, then $W$ reduces to the concircular curvature tensor and if $a=1$ and $b=-\frac{1}{(n-2)}$, then $W$ reduces to the conformal curvature tensor. Using the expression of the conformal and the concircular curvature tensor in (6.1), the quasi-conformal curvature tensor $W$ of type $(1,3)$ can be written as

$$
\begin{aligned}
W(X, Y) Z= & a R(X, Y) Z+b[S(Y, Z) X-S(X, Z) Y \\
& +g(Y, Z) Q X-g(X, Z) Q Y] \\
& -\frac{r}{n}\left(\frac{a}{n-1}+2 b\right)\{g(Y, Z) X-g(X, Z) Y\} .
\end{aligned}
$$

We now consider a $G(Q E)_{n}(n>3)$ satisfying the condition $W \cdot S=0$. Then we have

$$
S(W(X, Y) Z, U)+S(Z, W(X, Y) U)=0 .
$$

In view of (1.2), (6.3) yields

$$
\begin{aligned}
& \beta[A(W(X, Y) Z) A(U)+A(Z) A(W(X, Y) U)] \\
& +\gamma[B(W(X, Y) Z) B(U)+B(Z) B(W(X, Y) U)]=0 .
\end{aligned}
$$

Substituting $Z=\rho$ and $U=\mu$ in (6.4), we obtain

$$
(\gamma-\beta) W(X, Y, \rho, \mu)=0 .
$$

From (6.5), it follows that either $\beta=\gamma$ or

$$
W(X, Y, \rho, \mu)=0,
$$

which implies that

$$
\begin{aligned}
\text { (6.6) } a R(X, Y, \rho, \mu)= & -b[A(Q Y) B(X)-A(Q X) B(Y) \\
& +A(Y) B(Q X)-A(X) B(Q Y)] \\
& +\frac{r}{n}\left(\frac{a}{n-1}+2 b\right)\{A(Y) B(X)-A(X) B(Y)\} .
\end{aligned}
$$


Thus we can state the following:

Theorem 6.1. If a $G(Q E)_{n}(n>3)$ satisfies the condition $W \cdot S=0$, then either the associated scalars $\beta$ and $\gamma$ are equal or the curvature tensor $R$ of the manifold satisfies the property (6.6).

\section{7. $G(Q E)_{n}(n>3)$ satisfying the condition $P \cdot S=0$}

The Weyl projective curvature tensor $P$ of type $(1,3)$ of an $n$-dimensional Riemannian manifold $\left(M^{n}, g\right)(n>3)$ is defined by [3]

$$
P(X, Y) Z=R(X, Y) Z-\frac{1}{n-1}[S(Y, Z) X-S(X, Z) Y]
$$

for any vector fields $X, Y, Z \in \chi(M)$. Let us take a $G(Q E)_{n}(n>3)$ with $P \cdot S=0$. Then we get

$$
S(P(X, Y) Z, U)+S(Z, P(X, Y) U)=0 .
$$

Using (1.2) in (7.2), we get

$$
\begin{aligned}
& \alpha[\tilde{P}(X, Y, Z, U)+\tilde{P}(X, Y, U, Z)]+\beta[A(P(X, Y) Z) A(U) \\
& +A(Z) A(P(X, Y) U)]+\gamma[B(P(X, Y) Z) B(U) \\
& +B(Z) B(P(X, Y) U)]=0,
\end{aligned}
$$

where $\tilde{P}(X, Y, Z, U)=g(P(X, Y) Z, U)$. Setting $Z=\rho$ and $U=\mu$ in (7.3), we get

$$
(\alpha+\gamma) \tilde{P}(X, Y, \rho, \mu)+(\alpha+\beta) \tilde{P}(X, Y, \mu, \rho)=0 .
$$

In view of (7.1), we have from (7.4) that

$$
\begin{aligned}
& (n-1)(\gamma-\beta) R(X, Y, \rho, \mu) \\
=\quad & (\alpha+\gamma)\{A(Q Y) B(X)-A(Q X) B(Y)\} \\
+ & (\alpha+\beta)\{A(X) B(Q Y)-A(Y) B(Q X)\},
\end{aligned}
$$

provided $\gamma-\beta \neq 0$. This leads to the following:

Theorem 7.1. If a $G(Q E)_{n}(n>3)$ satisfies the condition $P \cdot S=0$, then the curvature tensor $R$ of the manifold satisfies the property (7.5), provided $\beta \neq \gamma$.

8. Conformally flat $G(Q E)_{n}(n>3)$ with $R(X, Y) \cdot S=0$

Let us consider a conformally flat $G(Q E)_{n}(n>3)$. Then, from (4.1), we get

$$
\begin{aligned}
R(X, Y) Z= & \frac{1}{n-2}[S(Y, Z) X-S(X, Z) Y \\
& +g(Y, Z) Q X-g(X, Z) Q Y] \\
& -\frac{r}{(n-1)(n-2)}\{g(Y, Z) X-g(X, Z) Y\} .
\end{aligned}
$$

Since the manifold satisfies $R(X, Y) \cdot S=0$, we get

$$
S(R(X, Y) Z, U)+S(Z, R(X, Y) U)=0 .
$$


Using (8.1) in (8.2), we get

$$
\begin{aligned}
& g(Y, Z) S(Q X, U)-g(X, Z) S(Q Y, U) \\
& +g(Y, U) S(Q X, Z)-g(X, U) S(Q Y, Z) \\
= & \frac{r}{n-1}[g(Y, Z) S(X, U)-g(X, Z) S(Y, U) \\
& +g(Y, U) S(X, Z)-g(X, U) S(Y, Z)] .
\end{aligned}
$$

Let $\lambda$ be the eigenvalue of the endomorphism $Q$ corresponding to an eigenvector $X$. Then $Q X=\lambda X$, i.e., $S(X, U)=\lambda g(X, U)$ and hence

$$
S(Q X, U)=\lambda S(X, U) \text {. }
$$

By virtue of (8.4), it follows from (8.3) that

$$
\begin{aligned}
& \left(\lambda-\frac{r}{n-1}\right)[g(Y, Z) S(X, U)-g(X, Z) S(Y, U) \\
& +g(Y, U) S(X, Z)-g(X, U) S(Y, Z)]=0,
\end{aligned}
$$

which yields

(8.5)

$$
g(Y, Z) S(X, U)-g(X, Z) S(Y, U)+g(Y, U) S(X, Z)-g(X, U) S(Y, Z)=0,
$$

provided $\lambda \neq \frac{r}{n-1}$. Again using (1.2) in (8.5), we get

$$
\begin{aligned}
& \beta[g(Y, Z) A(X) A(U)-g(X, Z) A(Y) A(U)+g(Y, U) A(X) A(Z) \\
& -g(X, U) A(Y) A(Z)]+\gamma[g(Y, Z) B(X) B(U)-g(X, Z) B(Y) B(U) \\
& +g(Y, U) B(X) B(Z)-g(X, U) B(Y) B(Z)]=0, \quad \text { provided } \lambda \neq \frac{r}{n-1} .
\end{aligned}
$$

Setting $Z=\rho$ and $U=\mu$, we get

$$
(\beta-\gamma)\{A(X) B(Y)-A(Y) B(X)\}=0 .
$$

From (8.7), we get either $\beta=\gamma$ or

$$
A(X) B(Y)=A(Y) B(X),
$$

that is, the vector fields $\rho$ and $\mu$ are co-directional. Thus we can state the following:

Theorem 8.1. If, in a conformally flat Ricci-semisymmetric $G(Q E)_{n}(n>3)$, $\frac{r}{n-1}$ is not an eigenvalue of the Ricci-operator $Q$, then either the associated scalars $\beta$ and $\gamma$ of the manifold are equal or the vector fields $\rho$ and $\mu$ corresponding to the 1-forms $A$ and $B$ respectively are co-directional.

\section{Some Examples of $G(Q E)_{n}$}

This section deals with several non-trivial examples of $G(Q E)_{n}$.

Example 9.1. We define a Riemannian metric $g$ on $\mathbb{R}^{4}$ by the formula (9.1)

$d s^{2}=g_{i j} d x^{i} d x^{j}=(1+2 p)\left[\left(d x^{1}\right)^{2}+\left(d x^{2}\right)^{2}+\left(d x^{3}\right)^{2}+\left(d x^{4}\right)^{2}\right],(i, j=1,2,3,4)$, 
where $p=\frac{e^{x^{1}}}{k^{2}}$ and $k$ is a non-zero constant. Then the only non-vanishing components of the Christoffel symbols, the curvature tensor, the Ricci tensor and scalar curvature are given by

$$
\begin{array}{r}
\Gamma_{22}^{1}=-\frac{p}{(1+2 p)}=\Gamma_{33}^{1}=\Gamma_{44}^{1}=-\Gamma_{11}^{1}=-\Gamma_{12}^{2}=-\Gamma_{13}^{3}=-\Gamma_{14}^{4}, \\
R_{1221}=R_{1331}=R_{1441}=\frac{p}{(1+2 p)}, S_{11}=\frac{3 p}{(1+2 p)^{2}}, \\
S_{22}=S_{33}=S_{44}=\frac{p}{(1+2 p)^{2}}, r=\frac{6 p}{(1+2 p)^{3}} \neq 0
\end{array}
$$

and the components which can be obtained from these by the symmetry properties.

Therefore $\mathbb{R}^{4}$ is a Riemannian manifold $\left(M^{4}, g\right)$ of non-vanishing scalar curvature. We shall now show that $M^{4}$ is a $G(Q E)_{4}$, i.e., it satisfies (1.2). Let us now consider the associated scalars as follows:

$$
\alpha=\frac{p}{(1+2 p)^{3}}, \beta=3 p, \gamma=-\frac{1}{(1+2 p)^{2}} .
$$

In terms of local coordinate system, let us consider the 1-forms $A$ and $B$ as follows:

$$
\begin{aligned}
& A_{i}(x)=\left\{\begin{array}{c}
\frac{1}{1+2 p} \text { for } i=1 \\
0 \text { otherwise }
\end{array}\right. \\
& B_{i}(x)=\left\{\begin{array}{c}
\sqrt{p} \text { for } i=1 \\
0 \text { otherwise }
\end{array}\right.
\end{aligned}
$$

In terms of local coordinate system, the defining condition (1.2) of a $G(Q E)_{n}$ can be written as

$$
S_{i j}=\alpha g_{i j}+\beta A_{i} A_{j}+\gamma B_{i} B_{j}, \quad i, j=1,2,3,4 .
$$

By virtue of (9.2) and (9.3), it can be easily shown that (9.4) holds for $i, j=$ $1,2,3,4$. Therefore $\left(M^{4}, g\right)$ is a $G(Q E)_{4}$, which is not quasi-Einstein. Hence we can state the following:

Theorem 9.1. Let $\left(M^{4}, g\right)$ be a Riemannian manifold endowed with the metric given in (9.1). Then $\left(M^{4}, g\right)$ is a $G(Q E)_{4}$ with non-vanishing scalar curvature which is not quasi-Einstein.

Example 9.2. We define a Riemannian metric $g$ on $\mathbb{R}^{4}$ by the formula

$$
d s^{2}=e^{2 x^{1}}\left(d x^{1}\right)^{2}+\sin ^{2} x^{1}\left[\left(d x^{2}\right)^{2}+\left(d x^{3}\right)^{2}+\left(d x^{4}\right)^{2}\right],
$$

where $0<x^{1}<\frac{\pi}{2}$ but $x^{1} \neq \frac{\pi}{4}$. Then the only non-vanishing components of the Christoffel symbols, the curvature tensor, the Ricci tensor and scalar curvature 
are

$$
\begin{aligned}
& \Gamma_{11}^{1}=1, \Gamma_{12}^{2}=\Gamma_{13}^{3}=\Gamma_{14}^{4}=\cot x^{1} \\
& \Gamma_{22}^{1}=-\frac{\sin 2 x^{1}}{2 e^{2 x^{1}}}=\Gamma_{33}^{1}=\Gamma_{44}^{1}, \\
& R_{1221}=-\sin ^{2} x^{1}\left(1+\cot x^{1}\right)=R_{1331}=R_{1441}, \\
& R_{2332}=\frac{\sin ^{2} x^{1} \cos ^{2} x^{1}}{e^{2 x^{1}}}=R_{2442}=R_{3443}, \\
& S_{22}=\frac{2 \cos ^{2} x^{1}-\sin ^{2} x^{1}\left(1+\cot x^{1}\right)}{e^{2 x^{1}}}=S_{33}=S_{44}, \\
& S_{11}=-3\left(1+\cot x^{1}\right), r=\frac{6\left(\cot ^{2} x^{1}-\cot x^{1}-1\right)}{e^{2 x^{1}}} \neq 0,
\end{aligned}
$$

provided $\left(\cot ^{2} x^{1}-\cot x^{1}-1\right) \neq 0$ and the components which can be obtained from these by the symmetry properties. Therefore $\mathbb{R}^{4}$ with the considered metric is a Riemannian manifold $\left(M^{4}, g\right)$ of non-vanishing scalar curvature. We shall now show that this $M^{4}$ is a $G(Q E)_{4}$, i.e., it satisfies (1.2). Let us now consider the associated scalars as follows:

$$
\alpha=\frac{2 \cot ^{2} x^{1}-\cot x^{1}-1}{e^{2 x^{1}}}, \beta=-\left(1+\cot x^{1}\right), \gamma=-2 \cot x^{1} .
$$

In terms of local coordinate system, let us consider the 1-forms $A$ and $B$ as follows:

$$
\begin{gathered}
A_{i}(x)=\left\{\begin{array}{c}
\sqrt{2} \text { for } i=1, \\
0 \text { otherwise, }
\end{array}\right. \\
B_{i}(x)=\left\{\begin{array}{c}
\sqrt{\cot x^{1}} \text { for } i=1, \\
0 \text { otherwise. }
\end{array}\right.
\end{gathered}
$$

In terms of local coordinate system, the defining condition (1.2) of a $G(Q E)_{n}$ can be written as (9.4). By virtue of (9.6) and (9.7), it can be easily shown that (9.4) holds for $i, j=1,2,3,4$. Therefore $\left(M^{4}, g\right)$ is a $G(Q E)_{4}$, which is not quasi-Einstein. Hence we can state the following:

Theorem 9.2. Let $\left(M^{4}, g\right)$ be a Riemannian manifold endowed with the metric given in (9.5). Then $\left(M^{4}, g\right)$ is a $G(Q E)_{4}$ with non-vanishing scalar curvature which is not quasi-Einstein.

Acknowledgement. The authors wish to express their sincere thanks and gratitude to the referee for his valuable suggestions towards the improvement of the paper. The first author (A. A. Shaikh) gratefully acknowledges the financial support of CSIR, New Delhi, India [Project F. No. 25(0171)/09/EMR-II] for persuing the revised version of the manuscript. 


\section{References}

[1] M. C. Chaki and R. K. Maity, On quasi-Einstein manifolds, Publ. Math. Debrecen 57 (2000), 297-306.

[2] U. C. De and G. C. Ghosh, On generalized quasi-Einstein manifolds, Kyungpook Math. J. 44 (2004), 607-615.

[3] U. C. De and A. A. Shaikh, Differential Geometry of Manifolds, Narosa Publishing House Pvt. Ltd., New Delhi, 2007.

[4] R. Deszcz, On pseudosymmetric spaces, Bull. Soc. Math. Belg. Sér. A, 44 (1992), no. 1, $1-34$

[5] K. Yano and S. Sawaki, Riemannian manifolds admitting a conformal transformation group, J. Diff. Geom. 2 (1968), 161-184.

Absos Ali Shaikh

Department of Mathematics

UNIVERSITY OF BURDWAN

BURDWAN - 713104

West Bengal, IndiA

E-mail address: aask20030yahoo.co.in

Shyamal Kumar Hui

Department of Mathematics

UNIVERSITY OF BURDWAN

BURDWAN - 713104

West Bengal, India 